International Journal of Applied Mathematics

Volume 33 No. $4 \quad 2020,573-583$

ISSN: 1311-1728 (printed version); ISSN: 1314-8060 (on-line version)

doi: http://dx.doi.org/10.12732/ijam.v33i4.2

\title{
CONTROLLABILITY OF THE BURGERS EQUATION UNDER THE INFLUENCE OF IMPULSES, DELAY AND NONLOCAL CONDITIONS
}

\author{
Cosme Duque ${ }^{1}$, Jahnett Uzcátegui ${ }^{2}$, \\ Hugo Leiva ${ }^{3} \S$, Oscar Camacho ${ }^{4}$ \\ 1,2 Universidad de Los Andes \\ Departamento de Matemáticas \\ Mérida - 5101 - VENEZUELA \\ ${ }^{3}$ Universidad Yachay Tech \\ San Miguel de Urcuqui, Imbabura - Ecuador \\ Ibarra - 100150 - ECUADOR \\ ${ }^{4}$ Escuela Politécnica Nacional, Quito, Ecuador \\ Quito - 170109 - ECUADOR
}

\begin{abstract}
In the case of the Burges equation, this work proves the following conjecture: impulses, delays, and nonlocal conditions, under some assumptions, do not destroy some posed system qualitative properties since they are themselves intrinsic to it. we verified that the property of controllability is robust under this type of disturbances. Specifically, we prove that the interior approximate controllability of the linear heat equation is not destroyed if we add impulses, nonlocal conditions, and a nonlinear perturbation with delay in the state. This is done by using new techniques avoiding fixed point theorems employed by A.E. Bashirov et al. In this case the delay helps us to prove the approximate controllability of this system by pulling back the control solution to a fixed curve in a short time interval, and from this position, we are able to reach a neighborhood of the final state in time $\tau$ by using the fact that the corresponding linear heat equation is approximately controllable on any interval $\left[t_{0}, \tau\right], 0<t_{0}<\tau$.
\end{abstract}

AMS Subject Classification: 93B05, 93C10

Received: January 15, 2020

(c) 2020 Academic Publications

$\S$ Correspondence author 
Key Words: interior approximate controllability; impulsive Burgers equation with delays and nonlocal conditions; strongly continuous semigroups; new technique

\section{Introduction}

In this paper, we prove the interior approximate controllability of the Burgers equation with delays, impulses, and nonlocal conditions; this is done by using new techniques avoiding fixed point theorems employed by A.E. Bashirov et al. $[3,4,5]$. In this case, the delay helps us to prove the approximate controllability by pulling back the control solution to a fixed curve in a short time interval, and from this position, we are able to reach a neighborhood of the final state in time $\tau$ by using the approximate controllability of the heat equation in any positive interval. In this regard, we proved the interior approximate controllability of the following semilinear PDE equation with delays, impulses, and nonlocal conditions, which represents a perturbation of the Burgers equation,

$$
\left\{\begin{array}{l}
\frac{\partial z}{\partial t}=\nu z_{x x}+1_{\omega} u(t)-z(t-r, x) z_{x}(t-r, x)+f(t, z(t-r), u(t), \\
z(t, 0)=z(t, 1)=0, \quad t \in[0, \tau] \\
z(s, x)-h\left(z\left(\tau_{1}+s, x\right), \ldots, z\left(\tau_{q}+s, x\right)\right)=\phi(s, x), \quad s \in[-r, 0] \\
z\left(t_{k}^{+}, x\right)=z\left(t_{k}^{-}, x\right)+I_{k}\left(t_{k}, z\left(t_{k}, x\right) u\left(t_{k}, x\right), x \in \Omega, k=1, \ldots, p\right.
\end{array}\right.
$$

where $\Omega=[0,1], \phi \in C\left([-r, 0] ; H_{0}^{1}\right), \omega$ is an open nonempty subset of $(0,1)$, $1_{\omega}$ denotes the characteristic function of the set $\omega$ and the distributed control $u$ belongs to $L_{2}\left([0, \tau] ; L_{2}[0,1]\right), \phi:[-r, 0] \times \Omega \longrightarrow \mathbb{R}$ is a continuous function and $0<\tau_{1}<\tau_{2}<\cdots<\tau_{q}<\tau$ and $0<t_{1}<\cdots<t_{p}<\tau$.

Our main hypotheses are that the nonlinear functions $f:[0, \tau] \times \mathbb{R} \longrightarrow \mathbb{R}$, $I_{k}:[0, \tau] \times \mathbb{R} \times \mathbb{R} \longrightarrow \mathbb{R}$ and $h: \mathbb{R}^{q} \longrightarrow \mathbb{R}$ are smooth enough such that the system (1) admits a unique mild solutions for each control $u$ according to $[15,16,17]$, and satisfy the following estimates for $z, w, u, v \in \mathbb{R}$ :

$$
\begin{gathered}
|f(t, z, u)-f(t, w, v)| \leq L\{|z-w|+|u-v|\}, \quad t \in[0, \tau], \\
\mid f\left(t, z, u|\leq a(t)| z \mid+b(t), \quad t \in[0, \tau], \quad z, u \in \mathbb{R}, \quad a(\cdot), b(\cdot) \in L_{\infty}[0, \tau],\right. \\
\left|h\left(z_{1}, \ldots, z_{q}\right)-h\left(w_{1}, \ldots, w_{q}\right)\right| \leq K \max _{1 \leq i \leq q}\left|z_{i}-w_{i}\right|, \quad z_{i}, w_{i} \in \mathbb{R} .
\end{gathered}
$$

We shall denote $Z=L_{2}(\Omega), Z^{1}=H_{0}^{1}(\Omega)$, and by $C$ the space of continuous functions

$$
C=\left\{\phi:[-r, 0] \longrightarrow Z^{1}: \phi \text { is continuous }\right\},
$$


endowed respectively with the norms,

$$
\begin{gathered}
\|\phi\|_{Z^{1}}=\sqrt{\|\phi\|_{Z}^{2}+\left\|\phi_{x}\right\|_{Z}^{2}}, \quad \phi \in Z^{1}, \\
\|\phi\|=\sup _{-r \leq s \leq 0}\|\phi(s)\|_{Z^{1}}, \text { and } \phi(s)(x)=\phi(s, x), \quad x \in \Omega .
\end{gathered}
$$

Also, we assume that:

$$
z\left(t_{k}, x\right)=z\left(t_{k}^{+}\right)=\lim _{t \rightarrow t_{k}^{+}} z(t, x), \quad z\left(t_{k}^{-}, x\right)=\lim _{t \rightarrow t_{k}^{-}} z(t, x)
$$

In this regard, we also consider the following natural Banach space to set evolution equations with delay, impulses and nonlocal conditions;

$$
\begin{aligned}
P C_{t_{1} . . t_{P}}([-r, \tau] ; Z)= & \left\{z: J=[-r, \tau] \rightarrow Z: z \in C\left(J^{\prime} ; Z\right), \exists z\left(t_{k}^{+}, \cdot\right),\right. \\
& \left.z\left(t_{k}, \cdot\right)=z\left(t_{k}^{+}, \cdot\right)\right\},
\end{aligned}
$$

where $J^{\prime}=[-r, \tau] \backslash\left\{t_{1}, \ldots, t_{p}\right\}$, endowed with the norm

$$
\|z\|_{P C}=\sup _{t \in[-r, \tau]}\|z(t, \cdot)\|_{Z}
$$

with

$$
\|z\|_{Z}=\left(\int_{\Omega}|z(x)|^{2} d x\right)^{1 / 2}, \quad z \in Z
$$

Definition 1. (Approximate Controllability) The system (1) is said to be approximately controllable on $[0, \tau]$ if for every $\phi \in C, z^{1} \in Z=U=L_{2}(\Omega)$ and $\varepsilon>0$, there exists $u \in C(0, \tau, U)$ such that the mild solution $z(t)$ of $(1)$ corresponding to $u$ verifies:

$$
z(0)+h\left(z_{\tau_{1}}, \ldots, z_{\tau_{q}}\right)(0)=\phi(0), \quad \text { and } \quad\left\|z(\tau)-z^{1}\right\|_{Z}<\varepsilon
$$

The main goal of this paper is to prove the following theorem:

Theorem 2. If the functions $f, I_{k}, h$ are smooth enough and satisfy the hypotheses (2)-(4), the system (1) is approximately controllable on $[0, \tau]$, for all $\tau>0$. 


\section{Abstract formulation of the problem}

In this section, we chose a Hilbert space where system (1) can be written as an abstract differential equation (see [10]); to this end, we consider the following notations: Let us consider the Hilbert space $Z=L^{2}(\Omega)$ and $0<\lambda_{1}<\lambda_{2}<$ $\cdots<\lambda_{j} \longrightarrow \infty$ the eigenvalues of operator $A \phi=-\nu \phi_{x x}$. Then, we have the following well known properties:

a) There exists a complete orthonormal set $\left\{\phi_{j}\right\}$ of eigenvectors of $A$.

b) For all $z \in D(A)$ we have

$$
A z=\sum_{j=1}^{\infty} \lambda_{j}\left\langle\xi, \phi_{j}\right\rangle \phi_{j}=\sum_{j=1}^{\infty} \lambda_{j} E_{j} z
$$

where $\langle\cdot, \cdot\rangle$ is the inner product in $Z$ and

$$
E_{n} z=\left\langle z, \phi_{j}\right\rangle \phi_{j} \text {. }
$$

So, $\left\{E_{j}\right\}$ is a family of complete orthonormal projections in $Z$ and $z=$ $\sum_{j=1}^{\infty} E_{j} z, z \in Z$.

c) $-A$ generates an analytic semigroup $\{T(t)\}$ given by

$$
T(t) z=\sum_{j=1}^{\infty} e^{-\lambda_{j} t} E_{j} z, \text { and }\|T(t)\| \leq e^{-\lambda_{1} t}, \quad t \geq 0 .
$$

Consequently, system (1) can be written as an abstract functional differential equations with impulses and nonlocal conditions in $Z$ :

$$
\left\{\begin{array}{l}
z^{\prime}=-A z+B_{\omega} u+f^{e}\left(t, z_{t}, u(t)\right), \quad z \in Z, \quad t \geq 0, \\
z(s)+g\left(z_{\tau_{1}}, \ldots, z_{\tau_{q}}\right)(s)=\phi(s), \quad s \in[-r, 0], \\
z\left(t_{k}^{+}\right)=z\left(t_{k}^{-}\right)+I_{k}^{e}\left(t_{k}, z\left(t_{k}\right), u\left(t_{k}\right)\right), \quad k=1,2, \ldots, p,
\end{array}\right.
$$

where $u \in L^{2}(0, \tau ; U), U=Z, B_{w}: U \longrightarrow Z, B_{\omega} u=1_{\omega} u$ is a bounded linear operator. $z_{t} \in C\left([-r, 0] ; H_{0}^{1}\right)$ and it is defined by $z_{t}(s)=z(t+s),-r \leq s \leq 0$ and the functions $I_{k}^{e}:[0, \tau] \times Z \times U \longrightarrow Z, f^{e}:[0, \tau] \times C \times U \longrightarrow Z$ and $g: C^{q}([-r, 0] ; Z \longrightarrow C([-r, 0] ; Z)$ are defined by

$$
\begin{gathered}
I_{k}^{e}(t, z, u)(x)=I_{k}(t, z(x), u(x)), \quad k=1, \ldots, p, \quad x \in \Omega, \\
f^{e}(t, \phi, u)(x)=\phi(-r, x) \phi_{x}(-r, x)+f(t, \phi(-r, x) u(x)), \quad x \in \Omega
\end{gathered}
$$

and

$$
\left.g\left(\phi_{1}, \ldots, \phi_{q}\right)\right)(s, x)=h\left(\phi_{1}(s, x), \ldots \phi_{q}(s, x) \quad \text { in }[-r, 0] \times \Omega .\right.
$$


Proposition 3. Under the conditions (2)-(4) the functions $f^{e}$ and $g$ satisfies for all $z, w \in P C_{t_{1} . . t_{P}}$ :

$$
\begin{gathered}
\left\|f^{e}(t, \phi, u)\right\| \leq\|\phi\|_{C}^{2}+4\|a\|_{L_{\infty}}+4\|b\|_{L_{\infty}} \sqrt{\mu(\Omega)} \\
\left\|f^{e}\left(t, \phi_{1}, u\right)-f^{e}\left(t, \phi_{2}, u\right)\right\| \leq\left\{\left\|\phi_{1}-\phi_{2}\right\|_{C}+L\right\}\left\|\phi_{1}-\phi_{2}\right\|_{C}, \\
\left\|g\left(z_{\tau_{1}}, \ldots, z_{\tau_{q}}\right)-g\left(w_{\tau_{1}}, \ldots, w_{\tau_{q}}\right)\right\| \leq K\|z-w\|_{P C([-r, \tau] ; Z)} .
\end{gathered}
$$

\section{Controllability of the linear equation}

In this section we shall present some characterization of the approximate controllability of the corresponding linear heat equation. To this end, we note that, for all $z_{0} \in Z$ and $u \in L^{2}(0, \tau ; U)$ the initial value problem

$$
\left\{\begin{array}{l}
z^{\prime}=-A z+B_{\omega} u(t), \quad z \in Z, \\
z\left(t_{0}\right)=z_{0}
\end{array}\right.
$$

admits only one mild solution defined, for all $t \in\left[t_{0}, \tau\right]$, with $0 \leq t_{0} \leq \tau$, by

$$
z(t)=z\left(t, t_{0}, z_{0}, u\right)=T(t) z_{0}+\int_{t_{0}}^{t} T(t-s) B_{\omega} u(s) d s .
$$

Definition 4. Corresponding with (12), we define the following operators: The controllability operator $\mathfrak{G}_{\tau \delta}: L^{2}(\tau-\delta, \tau ; U) \longrightarrow Z$ defined by

$$
\mathfrak{G}_{\tau \delta} u=\int_{\tau-\delta}^{\tau} T(\tau-s) B_{\omega} u(s) d s, \quad u \in L^{2}(\tau-\delta, \tau ; U),
$$

and adjoint of this operator $\mathfrak{G}_{\tau \delta}^{*}: Z \longrightarrow L^{2}(\tau-\delta, \tau ; U)$ is given by

$$
\left(\mathfrak{G}_{\tau \delta}^{*} z\right)(t)=B_{\omega}^{*} T^{*}(\tau-t) z, \quad t \in[\tau-\delta, \tau] .
$$

The Grammian controllability operator is given by:

$$
Q_{\tau \delta}=\mathfrak{G}_{\tau \delta} \mathfrak{G}_{\tau \delta}^{*}=\int_{\tau-\delta}^{\tau} T(\tau-t) B_{\omega} B_{\omega}^{*} T^{*}(\tau-t) d t .
$$

The following lemma holds in general for a linear bounded operator $G$ : $W \rightarrow Z$ between Hilbert spaces $W$ and $Z$ (see [6],[7],[8],[9] and [14]). 
Lemma 5. The following statements are equivalent to the approximate controllability of the linear system (12) on $[\tau-\delta, \tau]$ :

a) $\overline{\operatorname{Rang}\left(\mathfrak{G}_{\tau \delta}\right)}=Z$.

b) $\operatorname{Ker}\left(\mathfrak{G}_{\tau \delta}^{*}\right)=\{0\}$.

c) $\left\langle Q_{\tau \delta} z, z\right\rangle>0, z \neq 0$ in $Z$.

d) $\lim _{\alpha \rightarrow 0^{+}} \alpha\left(\alpha I+Q_{\tau \delta}\right)^{-1} z=0$.

e) For all $z \in Z$ we have $\mathfrak{G}_{\tau \delta} u_{\alpha}=z-\alpha\left(\alpha I+Q_{\tau \delta}\right)^{-1} z$, where

$$
u_{\alpha}=\mathfrak{G}_{\tau \delta}^{*}\left(\alpha I+Q_{\tau \delta}\right)^{-1} z, \quad \alpha \in(0,1] .
$$

So, $\lim _{\alpha \rightarrow 0^{+}} \mathfrak{G}_{\tau \delta} u_{\alpha}=z$ and the error $E_{\tau \delta} z$ of this approximation is given by the formula

$$
E_{\tau \delta} z=\alpha\left(\alpha I+Q_{\tau \delta}\right)^{-1} z, \quad \alpha \in(0,1] .
$$

Remark 6. The foregoing lemma implies that the family of linear operators $\Gamma_{\alpha \tau \delta}: Z \longrightarrow W$, defined for $0<\alpha \leq 1$ by

$$
\Gamma_{\alpha \tau \delta} z=\mathfrak{G}_{\tau \delta}^{*}\left(\alpha I+Q_{\tau \delta}\right)^{-1} z
$$

satisfies

$$
\lim _{\alpha \rightarrow 0^{+}} \mathfrak{G}_{\tau \delta} \Gamma_{\alpha \tau \delta}=I
$$

in the strong topology.

Lemma 7. $Q_{\tau \delta}>0$ if, and only if, the linear system (12) is controllable on $[\tau-\delta, \tau]$. Moreover, given an initial state $y_{0}$ and a final state $z^{1}$ we can find a sequence of controls $\left\{u_{\alpha}^{\delta}\right\}_{0<\alpha \leq 1} \subset L^{2}(\tau-\delta, \tau ; U)$

$$
u_{\alpha}=u_{\alpha, \delta}=\mathfrak{G}_{\tau \delta}^{*}\left(\alpha I+\mathfrak{G}_{\tau \delta} \mathfrak{G}_{\tau \delta}^{*}\right)^{-1}\left(z^{1}-T(\tau) y_{0}\right), \quad \alpha \in(0,1],
$$

such that the solutions $y(t)=y\left(t, \tau-\delta, y_{0}, u_{\alpha}\right)$ of the initial value problem

$$
\left\{\begin{array}{l}
y^{\prime}=A y+B_{\omega} u_{\alpha}(t), \quad y \in Z, \quad t>0 \\
y(\tau-\delta)=y_{0}
\end{array}\right.
$$

satisfies

$$
\lim _{\alpha \rightarrow 0^{+}} y\left(\tau, \tau-\delta, y_{0}, u_{\alpha}\right)=z^{1}
$$

Equivalently,

$$
\lim _{\alpha \rightarrow 0^{+}} y(\tau)=\lim _{\alpha \rightarrow 0^{+}}\left\{T(\delta) y_{0}+\int_{\tau-\delta}^{\tau} T(\tau-s) B_{\omega} u_{\alpha}(s) d s\right\}=z^{1} .
$$




\section{The system with impulses, delay and nonlocal conditions}

In this section, we shall prove the main result of this paper, the interior approximate controllability of the Burguers equation with impulses, delay and nonlocal conditions given by (1), which is equivalent to prove the approximate controllability of the system (8). In this regard, according to $[1,12,15,16,17]$, for all $\phi \in C$ and $u \in L^{2}(0, \tau ; U)$ the nonlocal Cauchy problem (8) admits only one mild solution $z \in P C_{t_{1} . . t_{P}}([-r, \tau] ; Z)$ given by

$$
\begin{aligned}
z(t) & =T(t) \phi(0)-T(t)\left[g\left(z_{\tau_{1}}, \ldots, z_{\tau_{q}}\right)(0)\right]+\int_{0}^{\tau} T(t-s) B_{\omega} u(s) d s \\
& +\int_{0}^{t} T(t-s) f^{e}\left(s, z_{s}, u(t)\right) d s \\
& +\sum_{0<t_{k}<t} T\left(t-t_{k}\right) I_{k}^{e}\left(t_{k}, z\left(t_{k}\right) u\left(t_{k}\right)\right), \quad t \in[0, \tau], \\
z(t) & +\left(g\left(z_{\tau_{1}}, \ldots, z_{\tau_{q}}\right)\right)(t)=\phi(t), \quad t \in[-r, 0] .
\end{aligned}
$$

Now, we are ready to prove the main result of this paper, which is the interior approximate controllability of Burgers equation with impulses, delays and nonlocal conditions.

\section{Proof of Theorem 2.}

Given $\phi \in C$, a final state $z^{1}$ and $\varepsilon>0$, we want to find a control $u_{\alpha}^{\delta} \in$ $L^{2}(0, \tau ; U)$ steering the system from $\phi(0)$ to an $\varepsilon$-neighborhood of $z^{1}$ on time $\tau$. Precisely, for $\alpha>0$ and $0<\delta<\min \left\{\tau-t_{p}, r\right\}$ small enough, there exists control $u_{\alpha}^{\delta} \in L^{2}(0, \tau ; U)$ such that corresponding of solutions $z^{\delta, \alpha}$ of (8) satisfies;

$$
\left\|z^{\delta, \alpha}(\tau)-z^{1}\right\| \leq \varepsilon
$$

In fact, we consider any $u \in L^{2}(0, \tau ; U)$ and the corresponding solution $z(t)=z(t, 0, \phi, u)$ of the Cauchy problem (8). For $\alpha \in(0,1]$, we define the control $u_{\alpha}^{\delta} \in L^{2}(0, \tau ; U)$ as follows

$$
u_{\alpha}^{\delta}(t)= \begin{cases}u(t), & \text { if } \quad 0 \leq t \leq \tau-\delta \\ u_{\alpha}(t), & \text { if } \quad \tau-\delta<t \leq \tau\end{cases}
$$

where

$$
u_{\alpha}(t)=B_{\omega}^{*} T^{*}(\tau-t)\left(\alpha I+\mathfrak{G}_{\tau \delta} \mathfrak{G}_{\tau \delta}^{*}\right)^{-1}\left(z^{1}-T(\delta) z(\tau-\delta)\right), \quad \tau-\delta<t \leq \tau .
$$


Since $0<\delta<\tau-t_{p}$, then $\tau-\delta>t_{p}$ and the corresponding solution $z^{\delta, \alpha}(t)=$ $z\left(t, 0, \phi, u_{\alpha}^{\delta}\right)$ of the nonlocal Cauchy problem (8) at time $\tau$ can be written as follows:

$$
\begin{aligned}
& z^{\delta, \alpha}(\tau)=T(\tau) \phi(0)-T(\tau)\left[g\left(z_{\tau_{1}}, \ldots, z_{\tau_{q}}\right)(0)\right]+\int_{0}^{\tau} T(\tau-s) B_{\omega}(s) d s \\
& +\int_{0}^{\tau} T(\tau-s) f^{e}\left(s, z_{s}^{\delta, \alpha}, u_{\alpha}^{\delta}(s)\right) d s \\
& +\sum_{0<t_{k}<\tau} T\left(\tau-t_{k}\right) I_{k}^{e}\left(t_{k}, z\left(t_{k}\right), u_{\alpha}^{\delta}\left(t_{k}\right)\right) \\
& =T(\delta)\left\{T(\tau-\delta) \phi(0)-T(\tau-\delta)\left[g\left(z_{\tau_{1}}, \ldots, z_{\tau_{1}}\right)(0)\right]\right. \\
& +\int_{0}^{\tau-\delta} T(\tau-\delta-s) B_{\omega}(s) u_{\alpha}^{\delta}(s) d s \\
& +\int_{0}^{\tau-\delta} T(\tau-\delta-s) f^{e}\left(s, z_{s}^{\delta, \alpha}, u_{\alpha}^{\delta}(s)\right) d s \\
& \left.+\sum_{0<t_{k}<\tau-\delta}^{\tau} T\left(\tau-\delta-t_{k}\right) I_{k}^{e}\left(t_{k}, z^{\delta, \alpha}\left(t_{k}\right), u_{\alpha}^{\delta}\left(t_{k}\right)\right)\right\} \\
& +\int_{\tau-\delta}^{\tau} T(\tau-s) B_{\omega} u_{\alpha}^{\delta}(s) d s+\int_{\tau-\delta}^{\tau} T(\tau-s) f^{e}\left(s, z_{s}^{\delta, \alpha}, u_{\alpha}^{\delta}(s)\right) d s \\
& \left.=T(\delta) z(\tau-\delta)+\int_{\tau-\delta}^{\tau} T(\tau-s) B_{\omega} u_{\alpha}(s)\right) d s \\
& +\int_{\tau-\delta}^{\tau} T(\tau-s) f^{e}\left(s, z_{s}^{\delta, \alpha}, u_{\alpha}(s)\right) d s .
\end{aligned}
$$

So,

$$
\begin{aligned}
z^{\delta, \alpha}(\tau) & =T(\delta) z(\tau-\delta)+\int_{\tau-\delta}^{\tau} T(\tau-s) B_{\omega} u_{\alpha}(s) d s \\
& +\int_{\tau-\delta}^{\tau} T(\tau-s) f^{e}\left(s, z_{s}^{\delta, \alpha}, u_{\alpha}(s)\right) d s
\end{aligned}
$$

The corresponding solution $y^{\delta, \alpha}(t)=y\left(t, \tau-\delta, z(\tau-\delta), u_{\alpha}\right)$ of the initial value problem (18) at time $\tau$ is given by:

$$
y^{\delta, \alpha}(\tau)=T(\delta) z(\tau-\delta)+\int_{\tau-\delta}^{\tau} T(\tau-s) B_{\omega} u_{\alpha}(s) d s .
$$

On the other hand, from Lemma 7 there exists $\alpha>0$ such that

$$
\left\|y_{\alpha}^{\delta}(\tau)-z^{1}\right\| \leq \varepsilon / 2 .
$$

Therefore, 


$$
\left\|z^{\delta, \alpha}(\tau)-y^{\delta, \alpha}(\tau)\right\| \leq \int_{\tau-\delta}^{\tau}\|T(\tau-s)\|\left\|f^{e}\left(s, z_{s}^{\delta, \alpha}, u_{\alpha}^{\delta}(s)\right)\right\| d s .
$$

Now, since $0<\delta<r$ and $\tau-\delta \leq s \leq \tau$, then $s-r \leq \tau-r<\tau-\delta$ and

$$
z^{\delta, \alpha}(s-r)=z(s-r)
$$

Hence, there exists $\delta$ small enough such that $0<\delta<\min \left\{r, \tau-t_{p}\right\}$ and

$$
\begin{gathered}
\left\|z^{\delta, \alpha}(\tau)-y^{\delta, \alpha}(\tau)\right\| \leq \int_{\tau-\delta}^{\tau}\|T(\tau-s)\|\left\|f^{e}\left(s, z_{s}, u_{\alpha}^{\delta}(s)\right)\right\| d s \\
\leq \int_{\tau-\delta}^{\tau}\|T(\tau-s)\|\left\{\left\|z_{s}\right\|^{2}+4\|a\|_{L_{\infty}}\left\|z_{s}\right\|+4\|b\|_{L_{\infty}} \sqrt{\mu(\Omega)}\right\} d s<\varepsilon / 2 .
\end{gathered}
$$

So,

$$
\begin{aligned}
\left\|z^{\delta, \alpha}(\tau)-z^{1}\right\| & \leq \int_{\tau-\delta}^{\tau}\|T(\tau-s)\|\left\|f^{e}\left(s, z_{s}, u_{\alpha}^{\delta}(s)\right)\right\| d s \\
& +\left\|y^{\delta, \alpha}(\tau)-z^{1}\right\| z<\varepsilon / 2+\varepsilon / 2=\varepsilon .
\end{aligned}
$$

This completes the proof of the theorem.

\section{References}

[1] H. Akca, A. Boucherif and V. Covachev, Impulsive functional-differential equations with nonlocal conditions, Internat. J. of Math. and Math. Sciences, 29, No 5 (2002), 251-256; DOI: 10.1155/S0161171202012887.

[2] A.E. Bashirov, N.I. Mahmudov, On controllability of deterministic and stochastic systems, SIAM J. of Control and Optimization, 37 (1999), 18081821.

[3] A.E. Bashirov and N. Ghahramanlou, On partial approximate controllability of semilinear systems, COGENTENG-Engeneering (2014); doi: 10.1080/23311916.2014.965947.

[4] A.E. Bashirov and M. Jneid, On partial complete controllability of semilinear systems. Abstract and Applied Analysis, 2013 (2013); doi: $10.1155 / 2013 / 521052$.

[5] A.E. Bashirov, N. Mahmudov, N. Semi and H. Etikan, On partial controllability concepts, Internat. J. of Control, 80, No 1 (Jan. 2007), 7 pp.; doi: 10.1080/00207170600885489. 
[6] A.E. Bashirov and N.I. Mahmudov, On controllability of deterministic and stochastic systems, SIAM J. on Control and Optimization, 37, No 6 (1999), 1808-1821.

[7] A.E. Bashirov and K.R. Kerimov, On controllability conception for stochastic systems, SIAM J. on Control and Optimization, 35, No 2 (1997), 384-398.

[8] R.F. Curtain and A.J. Pritchard, Infinite Dimensional Linear Systems, Lecture Notes in Control and Information Sciences, \# 8, Springer Verlag, Berlin (1978).

[9] R.F. Curtain and H.J. Zwart, An Introduction to Infinite Dimensional Linear Systems Theory, Text in Applied Mathematics, Springer Verlag, New York (1995).

[10] M.C. Delfour, S.K. Mitter, Controllability, observability and optimal feedback control of affine hereditary differential systems, SIAM J. on Control and Optimization, 10 (1972), 298-328.

[11] C. Guevara, H. Leiva, Controllability of the strongly damped impulsive semilinear wave equation with memory and delay, IMA J. of Mathematical Control and Information (2017) 00, 1011; doi: 10.1093/imamci/dnx042.

[12] R. Shikharchand Jain, M. Baburao Dhakne, On mild solutions of nonlocal semilinear impulsive functional integro-differential equations, Applied Mathematics. E-Notes, 13 (2013), 109-119.

[13] V. Lakshmikantham, D.D. Bainov and P.S. Simeonov, Theory of Impulsive Differential Equations, World Scientific, Singapore (1989).

[14] H. Leiva, N. Merentes and J. Sanchez, A characterization of semilinear dense range operators and applications, Abstract and Applied Analysis, 2013 (2013); doi: 10.1155/2013/729093.

[15] H. Leiva and P. Sundar, Approximate controllability of the burgers equation with impulses and delay, Far East J. of Math. Sciences, 102, No 10 (2017), 2291-2306; doi: 10.17654/MS102102291.

[16] H. Leiva, and P. Sundar, Existence of solutions for a class of semilinear evolution equations whit impulses and delays, J. of Nonlinear Evolution Equations and Applications 2017, No 7 (2017), 95108. 
[17] H. Leiva, Karakostas, Fixed point theorem and the existence of solutions for impulsive semilinear evolution equations with delays and nonlocal conditions, Communications in Mathematical Analysis, 21, No 2 (2018), 68-91. 
